\title{
Agravos na saúde Kaingáng (Terra Indígena Xapecó, Santa Catarina) e a estrutura dos serviços de atenção biomédica
}

\author{
Health problems among the Kaingáng \\ (Xapecó Indigenous Reserve, Santa Catarina) \\ and the health care system
}

Eliana E. Diehl 1

1 Departamento de Ciências

Farmacêuticas, Centro de Ciências da Saúde, Universidade Federal de Santa Catarina. Campus Universitário, Trindade, Florianópolis, SC 88040-900, Brasil. elianadiehl@hotmail.com

\begin{abstract}
The second semester of 1999 was a transition period for the implementation of the Special Indigenous Health District on the Xapecó Indigenous Reserve in western Santa Catarina State. The health clinic in the main village provided treatment with a staff including a general practitioner/obstetrician, pediatrician, dentist, nurse, two nursing assistants, and four nursing technicians. This paper presents the preliminary results of research on the organization of these health care services, their use by the community, and the health/disease profile of the Kaingáng, using patient files as the source of information. In September 1999, a total of 222 Indians were treated (children and adults), 50.5\% of whom residing in the main village. Among the Indians ages 0 to 14 years, infectious and parasitic diseases were the most frequent, supporting the idea that the Kaingang have precarious sanitary and nutritional conditions. Use of the clinic by adults was more varied, since of the 116 who appeared for consultation, 27 were pregnant women (out of a total of 86 women). In addition, prescriptions were written up for children and adults in $85.0 \%$ and $81.8 \%$ of the consultations, respectively.
\end{abstract}

Key words Health Profile; Health Services; Kaingáng; South American Indians

Resumo O segundo semestre de 1999 foi caracterizado como um período transitório para a montagem do Distrito Sanitário Especial Indígena Interior Sul na Terra Indígena Xapecó, oeste do Estado de Santa Catarina. No Posto de Saúde da aldeia Sede prestavam atendimento um clínico geral/obstetra e um pediatra, um dentista, uma enfermeira, dois auxiliares de enfermagem e quatro atendentes de enfermagem. Aqui são apresentados os resultados preliminares de um estudo voltado para descrever, entre outros aspectos, a organização dos serviços de saúde, seu uso pela comunidade e o perfil saúdeldoença dos Kaingáng, investigado pela análise de prontuários. No mês de setembro de 1999, foram atendidos 222 índios (crianças e adultos), sendo 50,5\% residentes na aldeia Sede. Entre os índios de 0 a 14 anos, as doenças infecto-parasitárias foram as mais recorrentes, fortalecendo a idéia de que os Kaingáng vivem em condições precárias de saneamento e alimentação. A procura do serviço pelos adultos, por sua vez, reflete uma certa complexidade, já que das 116 pessoas atendidas, 27 eram gestantes, em um total de 86 mulheres. Além disso, foram emitidas prescrições para as crianças e para os adultos em $85 \%$ e 81,8\% dos atendimentos, respectivamente.

Palavras-chave Perfil de Saúde; Serviços de Saúde; Kaingáng; Índios Sul-Americanos 


\section{Introdução}

Este trabalho apresenta informações relativas ao perfil de saúde dos Kaingáng da Terra Indígena (TI) Xapecó, localizada em Santa Catarina, Brasil, as quais resultaram da análise dos prontuários médicos. São também feitas considerações acerca da utilização dos serviços de saúde por parte dessa comunidade.

Nos últimos anos, a responsabilidade pela atenção à saúde indígena no Brasil tem flutuado entre a Fundação Nacional do Índio (FUNAI), a Fundação Nacional de Saúde (FUNASA) e, recentemente, com a paulatina implantação do subsistema de atenção à saúde (Brasil, 1999a) que tem os Distritos Sanitários Especiais Indígenas (DSEI's) como base (Brasil, 1999b) -, diversas prefeituras e/ou Organizações Não Governamentais têm assumido a questão de prover os serviços. Algumas missões religiosas e universidades também vinham prestando cuidados de saúde às comunidades indígenas.

Em que pesem as grandes diferenças regionais no país e a heterogeneidade social, cultural e do perfil epidemiológico das inúmeras etnias, os serviços biomédicos têm sido disponibilizados de maneira descontextualizada dessas realidades, contribuindo mais para o aumento da dependência do que para a efetiva melhoria do estado geral de saúde dessas populações. Com a implantação dos DSEI's, pretende-se alcançar mudanças significativas nas políticas de saúde indígena e na gestão de projetos direcionados ao atendimento integral dessas populações, procurando efetivar um modelo diferenciado de atenção com base na especificidade de cada um dos diversos grupos.

Coimbra Jr. \& Santos (2000) chamaram a atenção para a falta de informações fidedignas a respeito de populações indígenas brasileiras nos bancos de dados oficiais, conferindo-lhes "uma danosa invisibilidade demográfica e epidemiológica" (Coimbra Jr. \& Santos, 2000:131). De maneira geral, os registros existentes no que tange às condições de saúde dos índios são referidos aos grupos que vivem nas regiões Amazônica e Centro-Oeste, relegando as comunidades das regiões sul, sudeste e nordeste a abismo ainda mais profundo. Porém, se os dados sobre saúde são poucos e esparsos, é marcada a ausência de referências concernentes à utilização dos serviços de atenção biomédica que lhes são dispensados.

\section{Contexto local e coleta de dados}

A TI Xapecó possui área de 15.286 hectares, distribuída entre os municípios de Ipuaçu e Entre Rios, no oeste de Santa Catarina, e é dividida em nove aldeias Kaingáng e um Guaraní. Na área localizada em Ipuaçu viviam 2.802 indivíduos em agosto-setembro de 1999. A aldeia Sede era a mais populosa, contando-se 848 pessoas (SIAB-Ipuaçu, 1999). No Município de Entre Rios, a população indígena era de 1.050 pessoas, das quais $90 \%$ eram Kaingáng e os restantes, Guaraní (SIAB-Entre Rios, 1999).

A segunda metade do ano de 1999 distinguiu-se como período transitório para a montagem do DSEI Interior Sul no oeste de Santa Catarina. Na TI Xapecó, o Posto de Saúde da aldeia Sede (mais conhecido como "Enfermaria") funcionava diariamente com profissionais disponíveis durante 24 horas. Faziam parte do quadro dois médicos (clínica geral e pediatria), um dentista, uma enfermeira, dois auxiliares de enfermagem, quatro atendentes de enfermagem e uma servente. O médico A, contratado pelo município de Ipuaçu, atendia preferencialmente gestantes e mulheres, em geral, nas tardes de terça-feira (13h às $17 \mathrm{~h}$ ), ao passo que os homens tinham primazia nas manhãs de quinta-feira (09h às 12h). O médico $\mathrm{B}$, responsável pelo Programa de Saúde da Família local (PSF), consultava crianças de 0 a 14 anos nas tardes de segunda-feira e sexta-feira (13h às 16h). Em janeiro de 2000, após a assinatura do convênio entre Ipuaçu e a FUNASA para a operacionalização do DSEI, o médico A e o dentista foram substituídos por outros dois profissionais, que passaram a prestar serviço 40 horas semanais na aldeia Sede. A “Enfermaria” presta atendimento basicamente aos índios da etnia Kaingáng (nenhum Guaraní foi atendido nesse local de setembro de 1999 a fevereiro de 2000, período do trabalho de campo).

Os dados de prontuários médicos foram coletados na "Enfermaria" durante o mês de setembro de 1999 e foram analisados independentemente por três médicos de diferentes especialidades que não conheciam a realidade dos Kaingáng, visando construir hipóteses diagnósticas que levassem à classificação das doenças segundo a Classificação Internacional de Doenças, 10a edição (CID, 1993). As condutas adotadas pelos médicos A e B não foram divulgadas aos três médicos. Mediante consenso entre as três opiniões, optou-se por classificar as doenças dentro dos grandes grupos, de modo a garantir maior veracidade aos achados, já que em muitos prontuários havia somente a descrição de alguns sinais e sintomas. Cada visita aos 
médicos recebeu pelo menos uma classificação na CID-10, ou seja, para algumas pessoas houve mais de uma inclusão ou mesmo a repetição de um grupo.

\section{Resultados}

No mês de setembro, o médico A realizou 121 atendimentos entre gestantes e adultos, em geral, na "Enfermaria" cuja média foi de 15,1 atendimentos por período (total de oito períodos) e 16 atendimentos corresponderam a reconsultas. Dessas 121 consultas, seis gestantes foram encaminhadas a exame pré-natal; outros exames foram requeridos para 18 índios; quatro pessoas foram encaminhadas para especialistas, uma para hospitalização e uma para Curitiba. Foram emitidas 99 prescrições (ou seja, o médico prescreveu medicamentos em $81,8 \%$ dos atendimentos). Além disso, o médico emitiu 20 prescrições a pedido de um dos atendentes de enfermagem sem a presença do usuário, tratando-se a maioria de Notificações de Receita B (oito Notificações) e de Receita de Controle Especial em duas vias (seis Receitas); três prescrições foram de contraceptivo hormonal e as três restantes, de outros medicamentos. Considerando os índios que foram atendidos e aqueles que somente receberam prescrição (total de 116 pessoas), as mulheres representaram $74,1 \%$ do total e os homens $25,9 \%$, com concentração na faixa etária entre 15 e 44 anos (Figura 1). Os índios que moravam na aldeia
Sede foram os que mais procuraram a "Enfermaria” $(44,8 \%)$, seguidos pelos índios residentes na aldeia Olaria $(15,5 \%)$ e na aldeia Água Branca (12,9\%); 13,8\% eram de local ignorado; também foram atendidos índios provenientes das aldeias Fazenda São José, Baixo Samburá, Serrano, Paiol de Barro e Pinhalzinho.

A análise dos prontuários do médico A levou à classificação em 15 grupos da CID-10, sendo que os grupos XXI (Fatores que influenciam o estado de saúde e o contato com os serviços de saúde - 25,2\%), XV (Gravidez, parto e puerpério $-15,7 \%$ ), X (Doenças do aparelho respiratório - 11,3\%), XIV (Doenças do aparelho geniturinário - 11,3\%) e XVIII (Sintomas, sinais e achados anormais de exames clínicos e de laboratório, não classificados em outra parte - 10,7\%) totalizaram $74,2 \%$ do conjunto total (Figura 2).

O médico B atendeu 106 crianças no mesmo período, perfazendo a média de 15 consultas por tarde (total de oito tardes) e encaminhando 11 crianças para exames, nove para especialistas e seis para internação; foram emitidas 102 prescrições (isto é, 85\% das consultas resultaram em prescrição). Os meninos representaram $54,7 \%$ e as meninas, $45,3 \%$; com menos de um ano ambos os gêneros somaram $24,5 \%$; de 1 a 4 anos, 51,9\%; de 5 a 9 anos, $15,1 \%$; e de 10 a 14 anos, 8,5\% (Figura 3). A maior parte das crianças trazidas ao médico residia na aldeia Sede $(56,6 \%)$, seguidas pelas que moravam nas aldeias Água Branca $(14,2 \%)$ e Olaria $(9,4 \%)$; da aldeia Serrano vieram para consulta quatro crianças $(3,8 \%)$ e as restantes eram pro-

Figura 1

Número de indivíduos por faixa etária e gênero atendidos pelo médico A. Setembro de 1999.

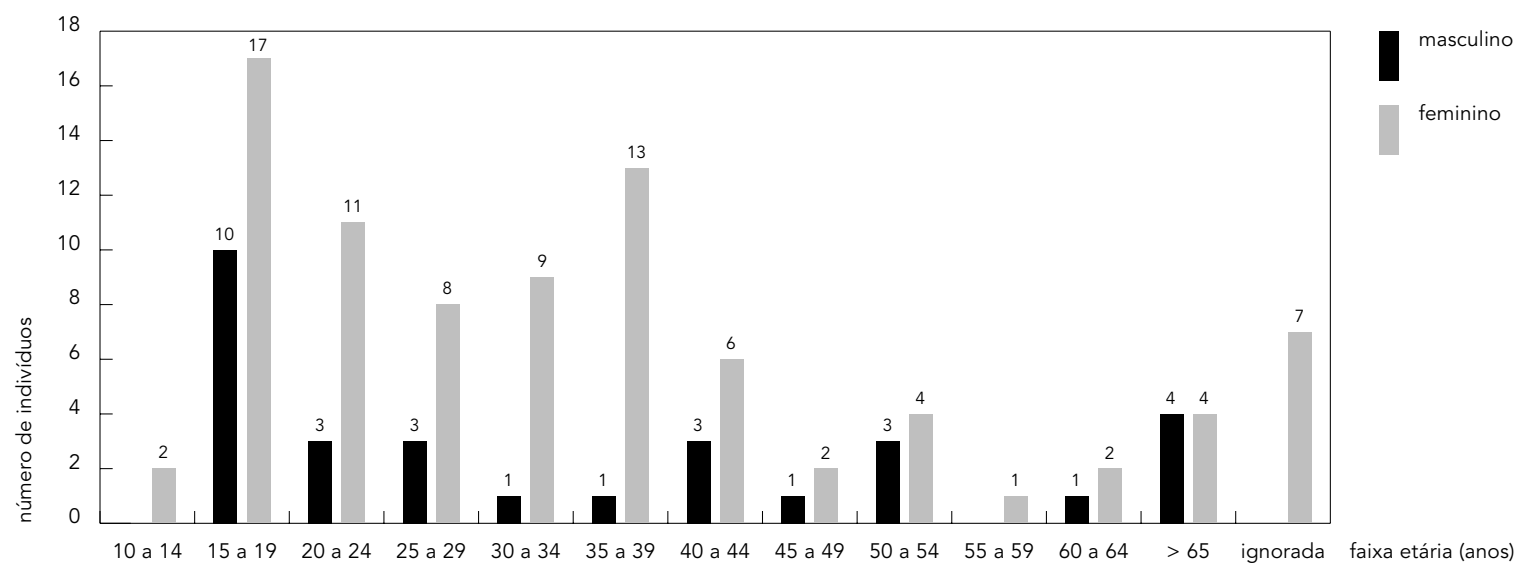


Figura 2

Classificação das morbidades nos grandes grupos (CID-10) para o médico A. Setembro de 1999.

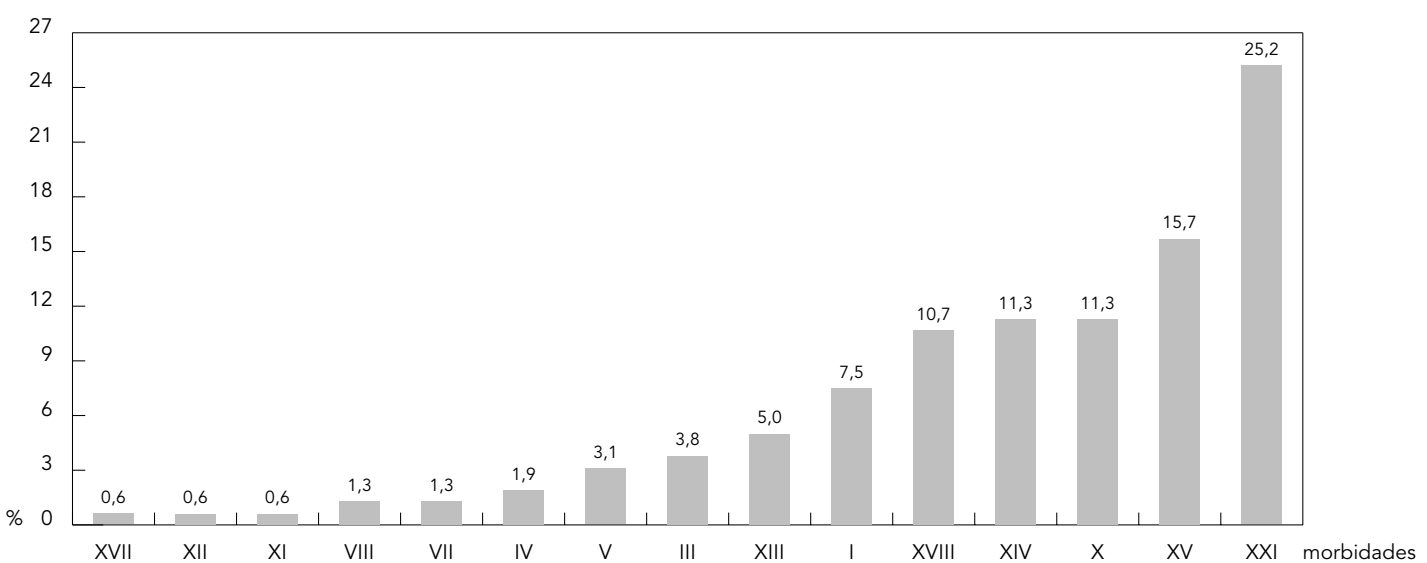

XVII: Malformações congênitas, deformidades e anomalias cromossômicas; XII: Doenças da pele e do tecido subcutâneo; XI: Doenças do aparelho digestivo; VIII: Doenças do ouvido e da apófise mastóide; VII: Doenças do olho e anexos; IV: Doenças endócrinas, nutricionais e metabólicas; V: Transtornos mentais e comportamentais; III: Doenças do sangue e dos órgãos hematopoéticos e alguns transtornos imunitários; XIII: Doenças do sistema osteomuscular e do tecido conjuntivo; I: Algumas doenças infecciosas e parasitárias; XVIII: Sintomas, sinais e achados anormais de exames clínicos e de laboratório, não classificados em outra parte; XIV: Doenças do aparelho geniturinário; X: Doenças do aparelho respiratório; XV: Gravidez, parto e puerpério; XXI: Fatores que influenciam o estado de saúde e o contato com os serviços de saúde.

Figura 3

Número de indivíduos por faixa etária e gênero atendidos pelo médico B.

Setembro de 1999

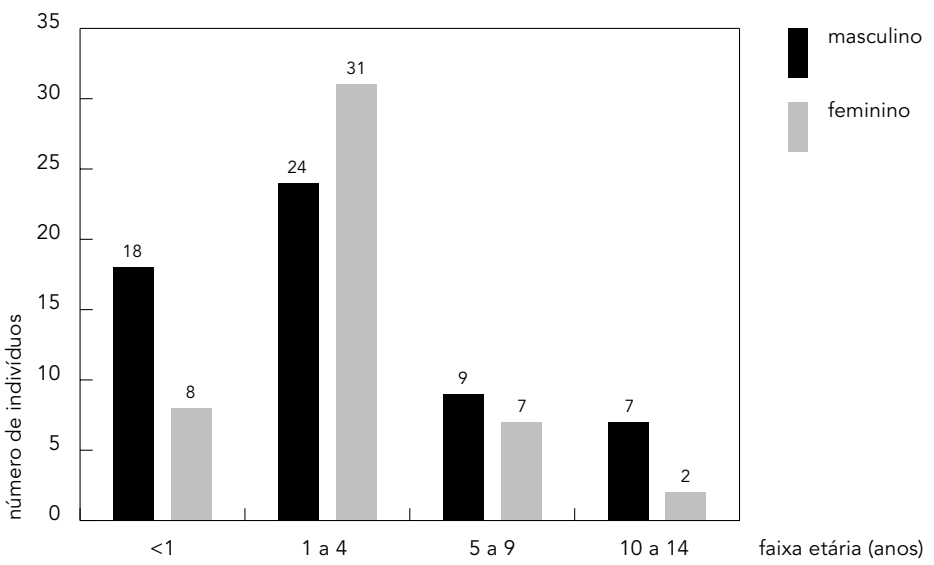

venientes das aldeias Fazenda, Pinhalzinho, Baixo Samburá e Barro Preto; 11,3\% vieram de local ignorado.

O exame dos prontuários do médico B permitiu classificar as morbidades em 15 grupos da CID-10. Os grupos I (Algumas doenças infecciosas e parasitárias - 43,1\%), X (Doenças do aparelho respiratório - 19,0\%), III (Doenças do sangue e dos órgãos hematopoéticos e alguns transtornos imunitários -6,3\%), XXI (Fatores que influenciam o estado de saúde e o contato com os serviços de saúde - 6,3\%) e XII (Doenças da pele e do tecido subcutâneo - 5,2\%) somaram $79,9 \%$ do quadro total (Figura 4).

As crianças e adultos residentes na aldeia Sede e atendidos por ambos os médicos no mês de setembro de 1999 representaram 13,2\% da população total dessa aldeia.

\section{Discussão}

A aldeia Sede, distante $24 \mathrm{~km}$ de Xanxerê, $23 \mathrm{~km}$ de Ipuaçu e $13 \mathrm{~km}$ de Entre Rios, é a que possui a melhor infra-estrutura, contando com uma escola estadual para ensino básico e médio, um Posto de Saúde (a "Enfermaria”), a administração local da TI (residência e "escritório" do cacique), um ginásio de esportes, uma igre- 
ja católica e duas igrejas evangélicas. Em outubro de 1999 foi iniciada a construção de um complexo educacional e cultural, composto de uma escola e um ginásio maiores do que os existentes, além de um centro cultural. À medida que na aldeia Sede foram sendo criadas condições favoráveis e facilidades, observou-se aumento da população. Além disso, o acesso facilitado contribuiu grandemente para torná-la a maior aldeia da TI, o que não significou tornála uma aldeia com melhor qualidade de vida. A maioria dos índios vive lá em moradias construídas precariamente. $\mathrm{O}$ acesso à energia elétrica e à água potável (de um poço artesiano) é restrito e não há sistema de esgoto; o lixo é deixado a céu aberto e, eventualmente, queimado. $\mathrm{Na}$ aldeia não há terra disponível para roças, motivo pelo qual algumas famílias se deslocam por quilômetros até os seus "sítios". Todavia, principalmente por questões políticas e econômicas internas, a maioria dos índios não dispõe de terra suficiente que garanta a sua subsistência, tornando-os dependentes do comércio local de alimentos. Ainda há que levar em conta o ecossistema bastante degradado, com pouquíssimos espécimes de mata nativa e de animais silvestres, assim como uma terra depauperada pelos arrendamentos a agroindústrias.
É nesse cenário que o serviço de saúde tem sido oferecido aos Kaingáng. Para os índios de 0 a 14 anos, que têm o maior percentual nas doenças de caráter infecto-parasitário, foi corroborada a idéia de que essa comunidade está inserida em uma realidade característica das populações pobres de outras regiões do Brasil, vivendo sob condições precárias, com alimentação protéico-calórica insuficiente, sem abastecimento de água potável e de rede e tratamento de esgotos. Os agravos respiratórios, que aparecem como segundo grupo mais freqüente nessa faixa etária, ainda podem ser explicados pelo fato de ter feito bastante frio em setembro de 1999, o que manteve os Kaingáng ao redor de seus fogos de chão e/ou fogões a lenha, cuja fumaça é mais um agravante para o quadro dessas doenças.

Os dados obtidos para os adultos a partir dos prontuários refletem a estrutura relativamente complexa do serviço. A presença de um clínico que atende uma vez por semana como obstetra, pode estar influenciando a grande procura por parte das índias grávidas. No universo de 116 pessoas atendidas em setembro, 27 eram gestantes, correspondendo a cerca de $31 \%$ sobre o número total de mulheres que consultaram. Além disso, as mulheres Kaingáng

Figura 4

Classificação das morbidades nos grandes grupos (CID-10) para o médico B. Setembro de 1999.

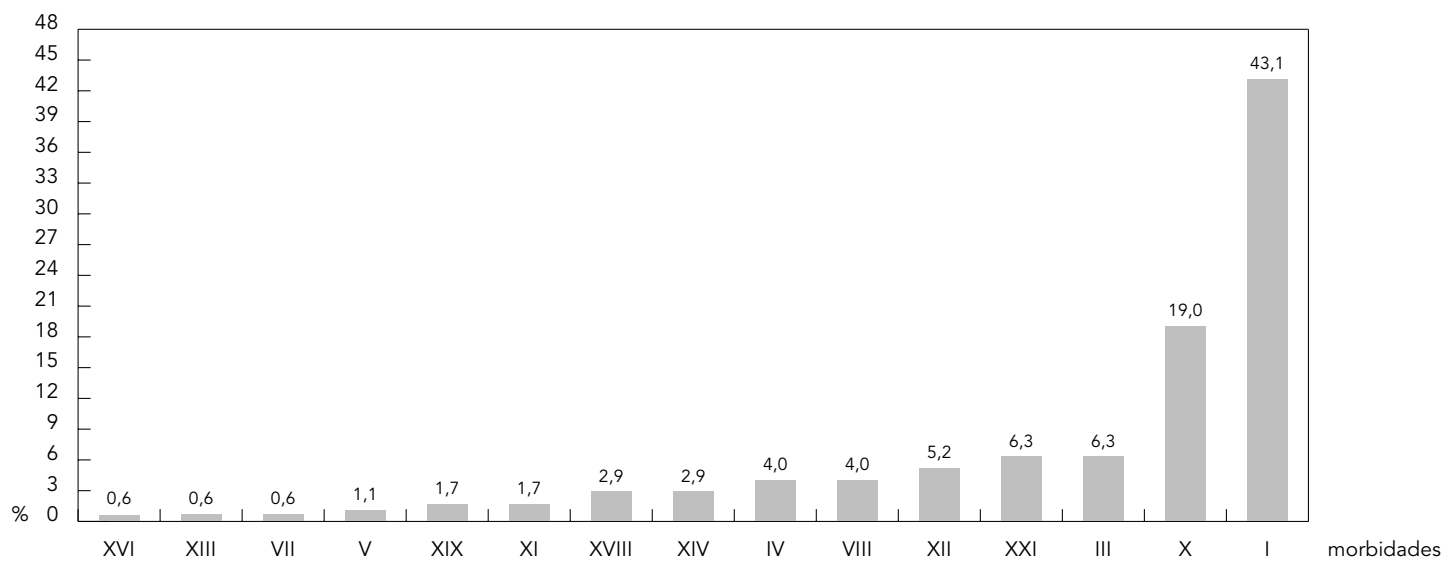

XVI: Algumas afecções originadas no período perinatal; XIII: Doenças do sistema osteomuscular e do tecido conjuntivo; VII: Doenças do olho e anexos; V: Transtornos mentais e comportamentais; XIX: Lesões, envenenamento e algumas outras conseqüências de causas externas; XI: Doenças do aparelho digestivo; XVIII: Sintomas, sinais e achados anormais de exames clínicos e de laboratório, não classificados em outra parte; XIV: Doenças do aparelho geniturinário; IV: Doenças endócrinas, nutricionais e metabólicas; VIII: Doenças do ouvido e da apófise mastóide; XII: Doenças da pele e do tecido subcutâneo; XXI: Fatores que influenciam o estado de saúde e o contato com os serviços de saúde; III: Doenças do sangue e dos órgãos hematopoéticos e alguns transtornos imunitários: X: Doenças do aparelho respiratório; I: Algumas doenças infecciosas e parasitárias. 
grávidas podem estar sendo estimuladas a ir ao serviço de saúde para serem inscritas no programa de Salário-Maternidade, benefício assegurado pela previdência social brasileira e que lhes garante um salário mínimo mensal por quatro meses. Se as gestantes forem excluídas do grupo, ainda assim as mulheres foram as que mais procuraram o serviço na "Enfermaria”, representando $66,3 \%$ de todos os atendimentos. Se considerarmos apenas o grupo XXI da CID-10, 29 mulheres e apenas sete homens foram influenciados pelo seu estado de saúde, o que os levou a procurar a "Enfermaria”. Segundo Green \& Pope (1999), o gênero feminino é um preditor para a alta utilização dos serviços médicos. Além disso, esses autores encontraram que o status de saúde, os sintomas físicos e mentais e os fatores psicossociais e comportamentais também são preditivos para o uso. Nesse sentido, Loyola (1984) observou entre moradores de um bairro do Município de Nova Iguaçu, Rio de Janeiro, que "a mulher é considerada o 'médico da família', cabendo-lhe não somente encaminhar os doentes aos serviços médicos mas também observar e conhecer sintomas, os remédios, as dietas e as alternativas de tratamento dos diferentes males que ocorrem no seio da família" (Loyola, 1984:128), situação semelhante à descrita por Queiroz (1993) ao analisar as representações e práticas sobre saúde e doença entre famílias operárias de dois bairros de Paulínea, São Paulo.

Entre os Kaingáng, foi observado que as mulheres são as principais responsáveis pelos diagnósticos primários e pelas tomadas de decisão a respeito do que fazer, o que as torna mais suscetíveis a procurar os serviços de saúde, os quais ainda suprem de modo gratuito quase que totalmente os medicamentos, afora a praticidade de estarem sendo oferecidos dentro da própria TI.

Nessa comunidade Kaingáng, tanto as crianças quanto os adultos têm sido amplamente tratados com medicamentos, como atestam as quantidades de prescrições emitidas por ambos profissionais, diferentemente dos achados de Haak (1988, 1989), os quais mostraram que em duas comunidades rurais na Bahia "as crianças são o foco central da cultura farmacêutica" (Haak, 1988:1424). A prática de medicina baseada eminentemente na prescrição e dispensação de medicamentos por parte dos médicos, como observado entre os Kaingáng, não contradiz a realidade verificada entre outros grupos sociais (Calva, 1996; Greenhalgh, 1987; Hardon, 1987; Paredes et al., 1996).

Além de todos esses fatores ligados às facilidades oferecidas na aldeia Sede, um elemento mais subjetivo pode estar concorrendo para a procura do serviço, citado algumas vezes por índios mais velhos (alguns deles curandeiros e um pajé), que é garantir primeiro o diagnóstico médico para só então buscar os recursos da medicina tradicional, evitando assim que os especialistas tradicionais carreguem culpa se, por acaso, acontecer algo de negativo com a pessoa doente.

É importante salientar que os dados de prontuário aqui analisados referem-se a registros feitos em um período de 30 dias, o que pode estar potencialmente mascarando situações que só se tornariam visíveis através de maior tempo de observação. Além disso, a "Enfermaria”, apesar de ser a referência central dentro dessa TI, não é o único local de recursos utilizado pelos Kaingáng, que buscam outros canais, sejam eles oficiais ou não, para a resolução de seus problemas. A falta de dados mais detalhados em alguns prontuários médicos também foi crítica na aproximação do perfil de saúde, já que a qualidade dessas informações estava comprometida. Outro fator a ser considerado diz respeito à baixa freqüência de uso dos serviços de saúde por parte dos homens, comparada à freqüência das mulheres, o que influenciou sobremaneira o perfil epidemiológico obtido para os adultos.

Os achados desse estudo, ainda que preliminares, sugerem que a possibilidade dada aos Kaingáng em relação às suas expectativas do modelo biomédico de atenção enfatiza sobretudo o que Lefèvre (1991) denomina "consumo de saúde" (consumo de consultas, exames, medicamentos e o restante das tecnologias biomédicas), com pouca ênfase na dimensão preventiva. Também é relevante mencionar que as condutas socializadas pela história de interação com os serviços de saúde (Cordeiro, 1985; Ross \& Duff, 1982) podem estar contribuindo para o delineamento dessa realidade.

O contexto apresentado revela o desafio que será, daqui para a frente, a efetiva implantação do DSEI com seu modelo diferenciado de saúde. Considerando a flutuabilidade das políticas e projetos de saúde no Brasil, em especial aqueles para os povos indígenas, que ora os colocam no centro das discussões e ora os legam ao esquecimento, é de se esperar que a criação de estrutura complexa não seja suficiente. A participação das comunidades indígenas em todos os níveis de discussão, de decisão e de operacionalização, a capacitação constante dos profissionais de saúde, a manutenção a longo prazo dos recursos, a implantação de sistema que forneça informações confiáveis aos bancos de dados oficiais, os investimentos em saneamento básico e moradias e a garantia de terra são alguns dos aspectos a serem também contemplados. 


\section{Agradecimentos}

Ao professor Ricardo V. Santos (Escola Nacional de Saúde Pública, Fiocruz) pelas importantes críticas e sugestões. À Fundação Coordenação de Aperfeiçoamento de Pessoal de Nível Superior (Bolsa PICD) e à Organização Pan-americana de Saúde, pelo financiamento desta pesquisa. Aos médicos que analisaram os prontuários dos pacientes indígenas.

\section{Referências}

BRASIL, 1999a. Lei n. 9.836 de 23 de Setembro de 1999. Acrescenta Dispositivos à Lei n. 8.080, de 19 de setembro de 1990, que "dispõe sobre as condições para a promoção, proteção e recuperação da saúde, a organização e o funcionamento dos serviços correspondentes e dá outras providências", instituindo o Subsistema de Atenção à Saúde Indígena. 11 de julho de 2000, <http://www.planalto. gov.br>.

BRASIL, 1999b. Decreto n. 3.156 de 27 de Agosto de 1999. Dispõe sobre as condições para a prestação de assistência à saúde dos povos indígenas, no âmbito do Sistema Único de Saúde, pelo Ministério da Saúde. Diário Oficial da União, Edição Extra. Brasília: I(165-A), pp. 37-38, 28 de agosto.

CALVA, J., 1996. Antibiotic use in a periurban community in Mexico: A household and drugstore survey. Social Science and Medicine, 42:11211128.

CID (Classificação Internacional de Doenças), 1993. Classificação Internacional de Doenças, $10 \underline{a}$ Ed. CD-ROM. São Paulo: Centro Brasileiro de Classificação de Doenças.

COIMBRA Jr., C. E. A. \& SANTOS, R. V., 2000. Saúde, minorias e desigualdades: Algumas teias de inter-relações, com ênfase nos povos indígenas no Brasil. Ciência \& Saúde Coletiva, 5:125-132.

CORDEIRO, H., 1985. A Indústria da Saúde no Brasil. 2a Ed. Rio de Janeiro: Graal.

GREEN, C. A. \& POPE, C. R., 1999. Gender, psychosocial factors and the use of medical services: A longitudinal analysis. Social Science and Medicine, 48:1363-1372.

GREENHALGH, T., 1987. Drug prescription and selfmedication in India: An exploratory survey. Social Science and Medicine, 25:307-318.

HAAK, H., 1988. Pharmaceuticals in two brazilian villages: Lay practices and perceptions. Social Science and Medicine, 27:1415-1427.

HAAK, H., 1989. Padrões de consumo de medicamentos em dois povoados da Bahia (Brasil). Revista de Saúde Pública, 23:143-151.
HARDON, A.P., 1987. The use of modern pharmaceuticals in a Filipino Village: Doctor's prescription and self medication. Social Science and Medicine, 25:277-292.

LEFÈVRE, F., 1991. O Medicamento como Mercadoria Simbólica. São Paulo: Editora Cortez.

LOYOLA, M.A., 1984. Médicos e Curandeiros - Conflito Social e Saúde. São Paulo: Difel.

PAREDES, P.; la PEÑA, M.; FLORES-GUERRA, E.; DIAZ, J. \& TROSTLE, J., 1996. Factors influencing physicians' prescribing behaviour in the treatment of childhood diarrhoea: knowledge may not be clue. Social Science and Medicine, 42:11411154.

QUEIROZ, M. S., 1993. Estratégias de consumo em saúde entre famílias trabalhadoras. Cadernos de Saúde Pública, 9:272-282.

ROSS, C. E. \& DUFF, R. S., 1982. Returning to the doctor: The efect of client characteristics, type of practice, and experiences with care. Journal of Health \& Social Behavior, 23:119-131.

SIAB-ENTRE RIOS (Sistema de Informação de Atenção Básica), 1999. Sistema de Informação de Atenção Básica. Entre Rios: Secretaria Municipal de Saúde.

SIAB-IPUAÇU (Sistema de Informação de Atenção Básica), 1999. Sistema de Informação de Atenção Básica. Ipuaçu: Secretaria Municipal de Saúde. 